\title{
A role for CCL28-CCR3 in T-cell homing to the human upper airway mucosa
}

\author{
E Danilova $^{1}$, I Skrindo ${ }^{1,2}$, E Gran $^{3}$, BJ Hales ${ }^{4}$, WA Smith ${ }^{4}$, J Jahnsen ${ }^{5}$, FE Johansen ${ }^{1,6}$, FL Jahnsen ${ }^{1}$ and \\ ES Baekkevold ${ }^{1}$
}

Lymphocyte recruitment to peripheral tissues is fundamental for immune surveillance and homeostasis, but the chemokines and chemokine receptors responsible for tissue-specific homing of Tcells to the upper airway mucosa have not been determined. To address this, we analyzed the chemokines expressed in the normal human nasal mucosa and found that CCL28 is preferentially expressed at a high level on the lumenal face of vascular endothelial cells in the mucosa. Analysis of the cognate chemokine receptors revealed that close to $50 \%$ of the CD4 ${ }^{+}$Tcells in the human nasal mucosa expressed the CCL28 receptor CCR3, whereas CCR3 was hardly detectable on Tcells in the small intestine and skin. In the circulation, $\mathrm{CCR}^{+}{ }^{+}$Tcells comprised a small subset that did not express homing receptors to the intestine or skin. Moreover, depletion of $\mathrm{CCR} 3^{+} \mathrm{CD} 4^{+} \mathrm{T}$ cells abrogated the proliferative response of human blood $\mathrm{CD} 4{ }^{+} \mathrm{T}$ cells against the opportunistic nasopharyngeal pathogen Haemophilus influenzae, indicating that the $\mathrm{CCR} 3^{+} \mathrm{CD} 4{ }^{+} \mathrm{T}_{\text {-cell }}$ subset in the circulation contains antigen specificities relevant for the upper airways. Together, these findings indicate that CCL28-CCR3 interactions are involved in the homeostatic trafficking of CD4 ${ }^{+} \mathrm{T}$ cells to the upper airways.

\section{INTRODUCTION}

Memory $\mathrm{T}$ cells are abundant within and beneath the epithelium in the airway mucosa. ${ }^{1}$ The concept of homeostatic tissue-specific homing to non-lymphoid tissues, in which memory lymphocytes preferentially traffic to the anatomical region where they first encountered their specific antigen, is well established. ${ }^{2}$ The tissue-tropic potential of lymphocytes appears to be imprinted during priming in regional lymphoid tissues, ${ }^{3}$ and the trafficking is mainly regulated at the level of cell recruitment from the blood and into the tissues. Lymphocyte recruitment to tissues involves a sequential process including chemokine-mediated control of intravascular lymphocyte adhesion. Studies have identified several constitutively expressed chemokines that control targeting of memory $\mathrm{T}$ cells to specific tissues. ${ }^{4}$ There is ample evidence to suggest that tissuespecific lymphocyte homing also occurs in the respiratory tract. This is particularly evident from numerous studies showing that plasma-cell precursors primed in the upper respiratory tract selectively migrate to the non-intestinal mucosa.,6
Moreover, recent animal studies have indicated that intranasal immunization induces $\mathrm{T}$ cells that are recruited to the airways under steady-state conditions. ${ }^{7,8}$

The best defined memory T-cell subsets targeted to specific extralymphoid sites are the skin-homing population, defined by expression of the cutaneous lymphocyte antigen (CLA), and the gut-homing population, defined by the high-level expression of the $\alpha 4 \beta 7$ integrin. ${ }^{9}$ Furthermore, it is well established that homing of CLA ${ }^{+} \mathrm{T}$ cells to cutaneous sites is mediated by CCL17-CCR4, ${ }^{10}$ a subset of which appear to also require CCL27-CCR10, ${ }^{11}$ whereas homing of $\alpha 4 \beta 7^{\text {hi }} \mathrm{T}$ cells to intestinal sites depend on CCL25-CCR9. ${ }^{12}$

Studies have also shown that memory $\mathrm{T}$ cells specific for pathogens exhibiting cutaneous or intestinal tropism are found within the circulating skin- and gut-homing populations, respectively. ${ }^{13,14}$ By analogy, non-typeable Haemophilus influenzae (NTHi) is an opportunistic pathogen normally colonizing the upper respiratory tract of children and adults that often cause infections such as otitis media, sinusitis, and

\footnotetext{
${ }^{1}$ Department of Pathology and Centre for Immune Regulation (CIR), Oslo University Hospital-Rikshospitalet and University of Oslo, Oslo, Norway. ${ }^{2}$ Department of Otorhinolaryngology, Akershus University Hospital, Nordbyhagen, Norway. ${ }^{3}$ Department of Otolaryngology, Lovisenberg Diakonale Hospital, Oslo, Norway. ${ }^{4}$ Centre for Child Health Research, University of Western Australia, Telethon Institute for Child Health Research, Subiaco, Western Australia, Australia. ${ }^{5}$ Department of Gastroenterology, Akershus

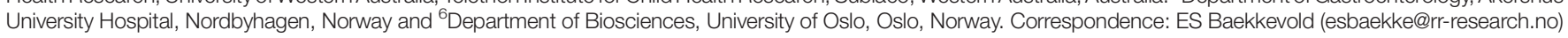


tonsillitis. ${ }^{15,16}$ NTHi-specific memory T cells circulate at low frequencies in the peripheral blood of healthy donors. ${ }^{17}$ The selective tissue localization of NTHi suggests that the immune responses to NTHi are associated with the generation of memory $\mathrm{T}$ cells trafficking to the upper airways.

The molecular mechanisms regulating the homeostatic migration of $\mathrm{T}$ cells to the human upper airway mucosa are not fully explored. In particular, identification of chemokines and the corresponding receptors controlling vascular recruitment is lacking. However, several reports have shown that CCL28 is preferentially expressed in various mucosal tissues of the upper aerodigestive tract. ${ }^{18,19}$ In fact, CCL28 may be assigned to a subfamily of evolutionary conserved chemokines, including CCL25 and CCL27, which are constitutively expressed at epithelial barrier surfaces and are implicated in steady-state lymphocyte homing to these sites. ${ }^{6,18}$ In this study, we found evidence that the CCL28 receptor CCR3 is involved in the steady-state trafficking of $\mathrm{CD} 4{ }^{+} \mathrm{T}$ cells to the human upper airway mucosa.

\section{RESULTS}

\section{Tissue chemokine expression profiles at steady state}

To quantify the tissue expression levels of chemokines in the nasal mucosa compared with the small intestinal mucosa and skin, we applied a real-time PCR array covering 39 different chemokines (Supplementary Table S1 online). To validate our approach, we first analyzed the expression of CCL25 as well as CCL27 and CCL17, which are constitutively expressed in the small intestinal mucosa and the skin, respectively ${ }^{3}$ (Figure 1a). Indeed, CCL25 and CCL27 were preferentially expressed at high levels in the small intestinal mucosa and the skin, respectively. Moreover, CCL17 transcripts were only detected in the skin samples. When comparing all chemokine transcripts expressed in the nasal mucosa with the skin and the intestine, we identified seven chemokines preferentially expressed in the nasal mucosa (Figure $\mathbf{1 b}$ and Supplementary Table S1). Among these, CCL28 and CXCL5 were found to exhibit the highest expression levels, and were subjected to further analysis.

\section{Vascular localization of CCL28 in the nasal mucosa}

To determine the protein expression and localization of chemokines in the nasal mucosa, we performed multicolor immunofluorescence stainings. Interestingly, CCL28 revealed a striking co-localization with the endothelial marker vWf in the lamina propria (Figure 2). In fact, the vast majority $(>80 \%)$ of $\mathrm{vWf}^{+}$vessels in the lamina propria coexpressed CCL28. Strong expression of CCL28 was detected on the luminal surface of the endothelial cells, as well as in perivascular cells, with an apparent gradual decrease in staining intensity within the surrounding tissue. Epithelial cells, particularly in the glands, were also strongly positive (data not shown). Immunostaining with a goat polyclonal anti-CCL28 antibody revealed a similar staining pattern (data not shown). CXCL5 appeared quite ubiquitously expressed within the lamina propria of the nasal mucosa (Figure 2). Although we found CXCL5 localized to regions surrounding vascular cells, the luminal enrichment of this chemokine was less apparent.

\section{CCL28 receptor expression on nasal mucosa-derived T cells}

Next we wanted to examine whether T cells in the nasal mucosa expressed the receptors for CCL28, CCR10, and CCR3. ${ }^{18,20}$ Tissue specimens of nasal mucosa were enzymatically digested, and single cell suspensions were analyzed by FACS. We found a distinct population of viable $\mathrm{CD} 3{ }^{+} \mathrm{CD} 4{ }^{+} \mathrm{T}$ cells that was readily detected among the dispersed cells (Figure 3a). Strikingly, whereas CCR10 expression was hardly detectable, approximately $50 \%$ of these nasal mucosa-derived $\mathrm{CD}^{+}$ $\mathrm{T}$ cells expressed CCR3 (Figure 3b). This strongly contrasted peripheral blood, in which CCR3 was expressed on a highly limited number of $\mathrm{CD}^{+} \mathrm{T}$ cells $(<1 \%)$. Furthermore, the $\mathrm{CD}^{+}{ }^{+} \mathrm{T}$-cell fraction from nasal mucosa contained approximately $20 \%$ CCR3 $^{+}$cells, (Figure 3c), and was also negative for CCR10. However, CCR10 expression was clearly detectable on a population of $\mathrm{CD} 138^{+}$plasma cells from the nasal mucosa (Supplementary Figure S1), as previously reported. ${ }^{5}$ A small percentage of the nasal mucosa-derived $\mathrm{CD}^{+} \mathrm{T}$ cells expressed the CXCL5 receptor CXCR1 (Figure 3d), but the level was similar to the reported expression level in peripheral blood. ${ }^{21}$ Expression of the closely related receptor CXCR2 was only observed on a minor fraction (Figure 3d). Interestingly, similar analysis performed on cells isolated from the skin and the small intestinal mucosa revealed very low numbers of
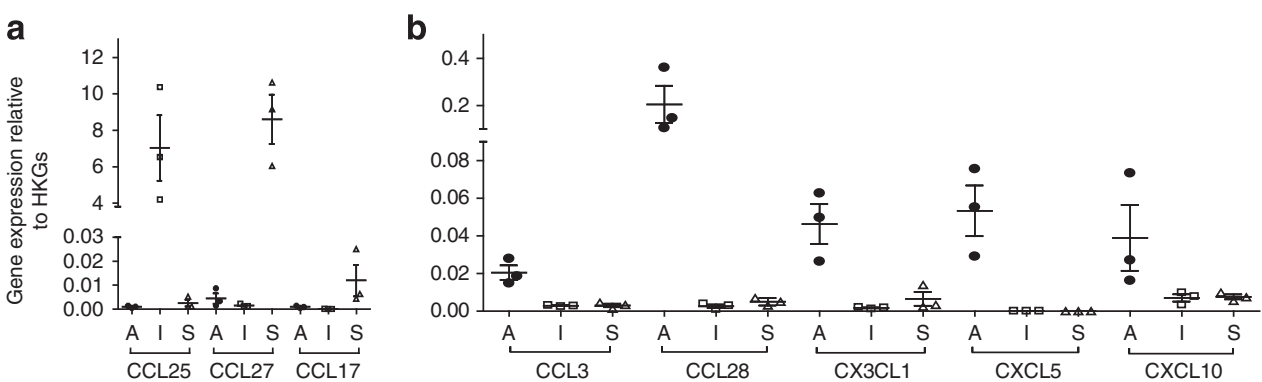

Figure 1 CCL28 is preferentially and highly expressed in the normal nasal mucosa. Chemokine gene expression was assessed by real-time reverse transcriptase-PCR on RNA isolated from tissue samples of upper airway (A) mucosa, small intestinal (I) mucosa, and skin (S). Genes preferentially expressed in small intestinal mucosa or skin (a), and genes preferentially expressed in nasal mucosa (b) are shown as expression relative to a collection of five housekeeping genes (HKGs). 


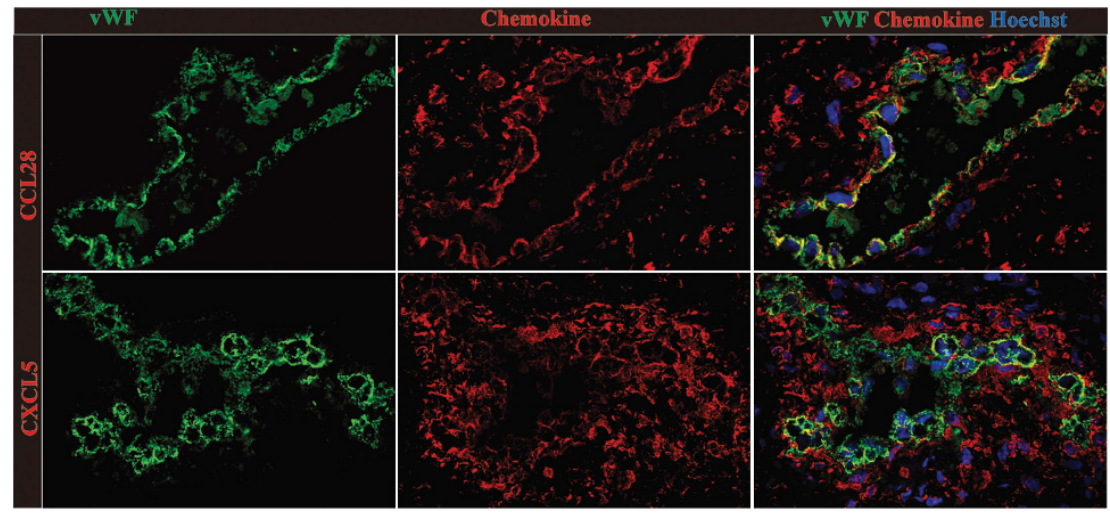

Figure 2 CCL28 is detected in the vascular lumen of nasal mucosal venules. Confocal images of immunuflourescence stainings for von Willebrand factor (vWF) (left), CCL28, and CXCL5 (middle) as well as images merged with the nuclear dye Hoechst (right) in cryosections of normal nasal mucosa. Images representative of nine donor biopsies analyzed. Original magnification $(\times 400)$.

a

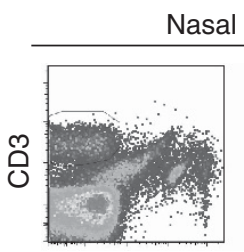

Propidium iodide

\section{b}

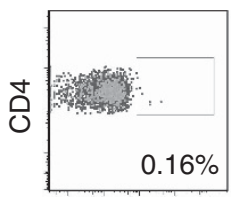

CCR10

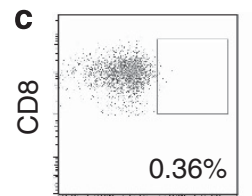

CCR10

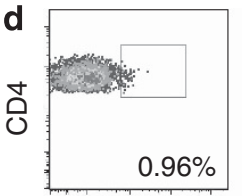

CXCR1 e
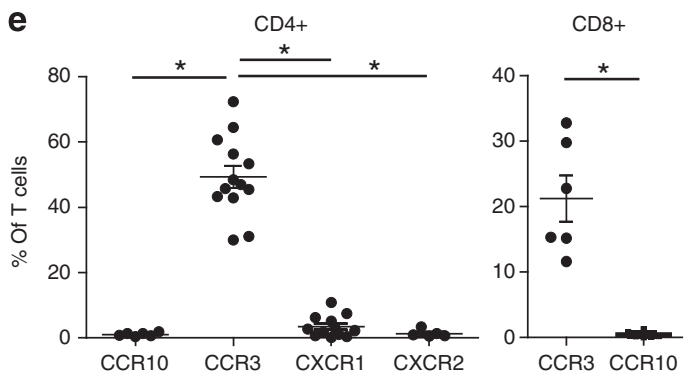

PBMC

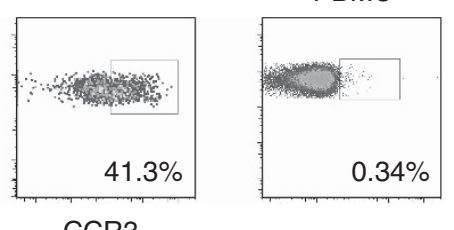

f

Skin

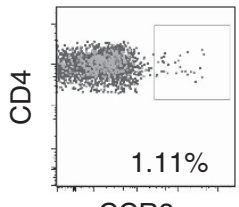

Intestine

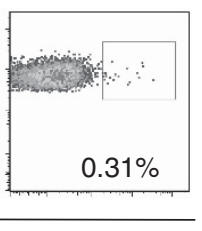

g

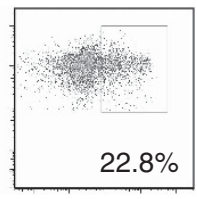

CCR3

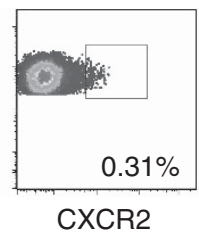

Figure $3 \mathrm{CCR}^{+} \mathrm{CD}^{+}$T cells are enriched in the normal nasal mucosa, but not in the skin and small intestine. (a) Samples of enzymatically digested nasal mucosa contained readily identifiable and viable CD3 ${ }^{+} \mathrm{CD} 4^{+}$T cells. Gating strategy and representative dot plots of $n>15$ donors is shown. (b-d) The percentage of chemokine receptor-positive $\mathrm{CD} 4^{+}$or $\mathrm{CD} 8^{+} \mathrm{CD} 3^{+} \mathrm{T}$ cells in samples from nasal mucosa (left and middle plots) and peripheral blood mononuclear cells (PBMCs; b, right plot as indicated). (e) Percentage of chemokine receptor-positive nasal mucosal T cells compiled from $n=10-14$ individual donors $\left({ }^{*} P<0.05\right)$. (f) CCR3 expression on $\mathrm{CD} 3^{+} \mathrm{CD} 4^{+} \mathrm{T}$ cells derived from enzymatically digested skin and small intestinal mucosa (as indicated). One representative of three individual donors is shown. (g) Expression of CCR3 and CLA (left) or CCR3 and $\alpha 4 \beta 7$ (right) on $\mathrm{CD}_{4}{ }^{+} \mathrm{T}$ cells from PBMCs. The percentage indicated shows the percentage of $\mathrm{CCR}^{+}$homing-receptor negative cells. One representative out of three experiments is shown. All gates were set based on irrelevant isotype controls.

$\mathrm{CCR}^{+}{ }^{+} \mathrm{CD} 4^{+} \mathrm{T}$ cells (Figure $3 \mathbf{f}$ ). $\mathrm{CD} 4^{+}$T cells expressing the homeostatic chemokine receptor CCR6 were generally scarce in the nasal mucosa (Supplementary Figure S3a). Moreover, mucosal $\mathrm{T}$ cells were mostly CCR7 ${ }^{-}$(Supplementary
Figure S3a), thus likely representing effector $\mathrm{T}$ cells with limited lymph node trafficking capacity.

In the circulation, CCR10 is expressed on a limited number of $\mathrm{CD} 4^{+} \mathrm{T}$ cells confined to a subpopulation of skin-homing 
$\mathrm{CLA}^{+} \mathrm{T}$ cells. ${ }^{11}$ Conversely, we found that $\mathrm{CCR} 3{ }^{+} \mathrm{CD} 4{ }^{+}$ $\mathrm{T}$ cells in peripheral blood $(<0.5 \%)$ were virtually negative for CLA (Figure 3g). Moreover, CCR $3^{+} \mathrm{CD} 4{ }^{+} \mathrm{T}$ cells appeared enriched within the $\alpha 4 \beta 7^{\mathrm{lo}}$ population, together indicating that these cells do not traffic to the skin or small intestine.

\section{Depletion of $\mathrm{CCR}^{+}{ }^{+} \mathrm{CD} 4{ }^{+} \mathrm{T}$ cells abrogates the proliferative $\mathrm{CD}^{+}{ }^{+} \mathrm{T}$-cell response to the NTHi-derived outer membrane protein P6}

The highly conserved outer membrane protein P6 from a range of NTHi isolates has been shown to induce T-cell responses. ${ }^{22}$ To assess the presence of $\mathrm{T}$ cells specific for NTHi within the $\mathrm{CCR}^{+}{ }^{+} \mathrm{CD} 4{ }^{+}$T-cell population, we determined the P6-specific proliferative response in purified peripheral blood $\mathrm{CD} 4^{+} \mathrm{T}$ cells undepleted or depleted for CCR $3^{+}$cells. To enable sufficient separation between the rare proliferating $\mathrm{T}$ cells and the nonresponding population, CFSE dilution in T cells was assessed after 6 days of stimulation. Analysis revealed that the subjects mounted a response to P6 following stimulation with antigen (Figure 4a), as published by others. ${ }^{17,22}$ Strikingly, when $\mathrm{CD} 4{ }^{+} \mathrm{T}$ cells were depleted for CCR $3^{+}$cells with FACS sorting, the proliferative response was abrogated and not different from unstimulated control cells. Similar results were also obtained by depleting $\mathrm{CD} 4{ }^{+} \mathrm{CCR} 3^{+}$cells with magnetic beads prior to antigen stimulation (data not shown), thus showing that the reduced proliferation was not an artifact introduced by cell passage through the FACS. On the contrary, depletion of $\mathrm{CD} 4{ }^{+} \mathrm{CCR} 3{ }^{+}$ cells in donors vaccinated with tetanus toxoid affected the tetanus toxoid memory response to a low extent (Figure 4a). To assess whether cytokine production by $\mathrm{P} 6$-stimulated $\mathrm{T}$ cells was altered by depletion of CCR3 $+\mathrm{CD} 4+\mathrm{T}$ cells, supernatants from the P6-stimulated cultures were analyzed. We found a significant reduction in the interleukin (IL)-2 levels following CCR3 depletion in the donors exhibiting proliferative responses (Figure 4c). However, levels of other cytokines analyzed, including IL-4, IL-5, IL-10, IL-13, and IFN- $\gamma$, were not significantly altered by depleting the $\mathrm{CCR} 3{ }^{+} \mathrm{CD} 4{ }^{+}$population (data not shown). However, when directly analyzing IFN- $\gamma$ production in P6-stimulated CD4 ${ }^{+}$T cells, we found that the vast majority of proliferating T cells were IFN- $\gamma^{+}$(Figure 4d). To determine whether the P6-specific $\mathrm{CD} 4^{+}$T-cell response was HLA class II restricted, PBMCs were stimulated with P6 for 6 days, with or without the addition of an anti-HLA class II antibody. Analysis of cultures from responding donors revealed that such treatment consistently reduced the proliferation to background levels observed in non-stimulated PBMCs (Figure 4e), thus strongly supporting that the P6-specific response is HLA class II dependent. To analyze the stimulatory effect of P6 directly on $\mathrm{CD} 4{ }^{+} \mathrm{CCR} 3^{+} \mathrm{T}$ cells, efforts were made to purify this subset through repeated rounds of positive selection. However, the low yield and reduced viability of the positively selected cell population precluded further analysis.

\section{DISCUSSION}

CCL28 belongs to a subfamily of evolutionary conserved chemokines, including CCL25 and CCL27, which are constitutively expressed at epithelial barrier surfaces and are implicated in steady-state lymphocyte homing to these sites. ${ }^{6,18}$ Our observation of high constitutive expression of CCL28 in the steady-state nasal mucosa is consistent with several other reports showing CCL28 expression in various mucosal tissues of the upper aerodigestive tract. ${ }^{18,19}$ In the nasal mucosa, CCL28 protein was readily detected on the luminal face of blood endothelial cells, which may indicate that endothelial cells produce this chemokine. However, it is well established that endothelial cells may constitutively transcytose extravascular chemokines to their lumen, ${ }^{23,24}$ thus the CCL28 may also be derived from other cells.

CCL28 is a ligand for CCR10 and CCR3, ${ }^{18,20}$ and CCL28CCR10 interactions have been implicated in the dissemination of antibody-secreting cells to the upper airway mucosa. In fact, plasmablasts induced in the secondary lymphoid organs of the upper airways express high levels of CCR10. ${ }^{5,25}$ However, we found that $\mathrm{T}$ cells residing in the nasal mucosa did not express CCR10. This receptor therefore appears to be restricted to plasma cells at this site. ${ }^{5}$ Strikingly, $\sim 50 \%$ of $\mathrm{CD} 4{ }^{+} \mathrm{T}$ cells from healthy nasal mucosa express the CCL28 receptor CCR3. This translates into a $\sim 100$-fold enrichment of this population compared with $\mathrm{CD}^{+}{ }^{+} \mathrm{T}$ cells in blood. No enrichment of CCR $3{ }^{+} \mathrm{CD} 4{ }^{+} \mathrm{T}$ cells was observed in the small intestinal mucosa or the skin. Furthermore, $\mathrm{CCR} 3{ }^{+} \mathrm{CD} 4{ }^{+} \mathrm{T}$ cells in peripheral blood exhibited low expression levels of the $\alpha 4 \beta 7$ integrin and CLA, indicating that circulating $\mathrm{CCR} 3{ }^{+} \mathrm{CD} 4{ }^{+}$ $\mathrm{T}$ cells do not home to the skin or the intestine.

CCR3 has several chemokine ligands ${ }^{20}$ that may potentially account for the tissue accumulation of this T-cell subset. The classical CCR3-ligand is CCL11, but we found this to be expressed at very low levels in the steady-state nasal mucosa (Supplementary Table S1). CCL5 mRNA was significantly expressed in our tissue material, but subsequent immunostainings did not reveal any vascular localization of the chemokine (data not shown). Moreover, transcripts for CCL7, CCL8, CCL13, and CCL15 were undetectable in the nasal mucosa. However, studies have shown that CCL28 is chemotactic for human eosinophils expressing CCR $3,{ }^{18}$ and CCL28-CCR3 interactions were found to regulate eosinophil recruitment independently of CCL11 to the airways in a mouse model of allergic airway inflammation. ${ }^{26}$ Unfortunately, the low frequency of CCR3 + T cells in peripheral blood precludes chemotaxis analysis of primary $\mathrm{T}$ cells with standard assays. However, we were able to detect rapid intracellular Ca flux specifically in CCR3 ${ }^{+} \mathrm{T}$ cells following CCL28 stimulation (Supplementary Figure S2).

Several studies have analyzed the chemokine expression in the upper airways during inflammation, and a role for the CCR4 ligands CCL17 and CCL22 appears important for T-cell recruitment, as both of these chemokines are strongly upregulated following allergen challenge in atopic subjects. ${ }^{27,28}$ Furthermore, a study revealed an increase of CCR $4^{+} \mathrm{T}$ cells in allergen-induced inflammation of the nasal mucosa in allergic rhinitis patients. ${ }^{29}$ However, we found that CCL17 and CCL22 (Figure 1) were expressed at very low levels at this site in the 

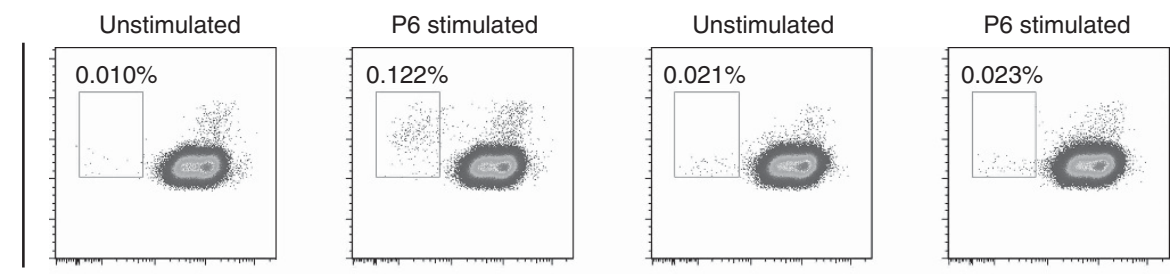

怘
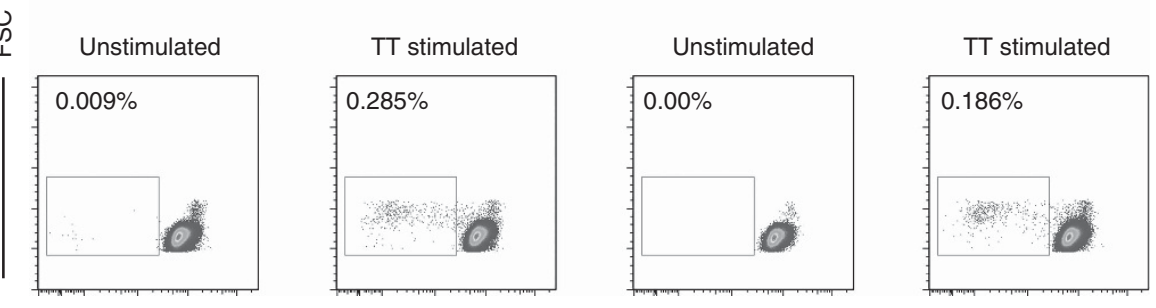

CFSE
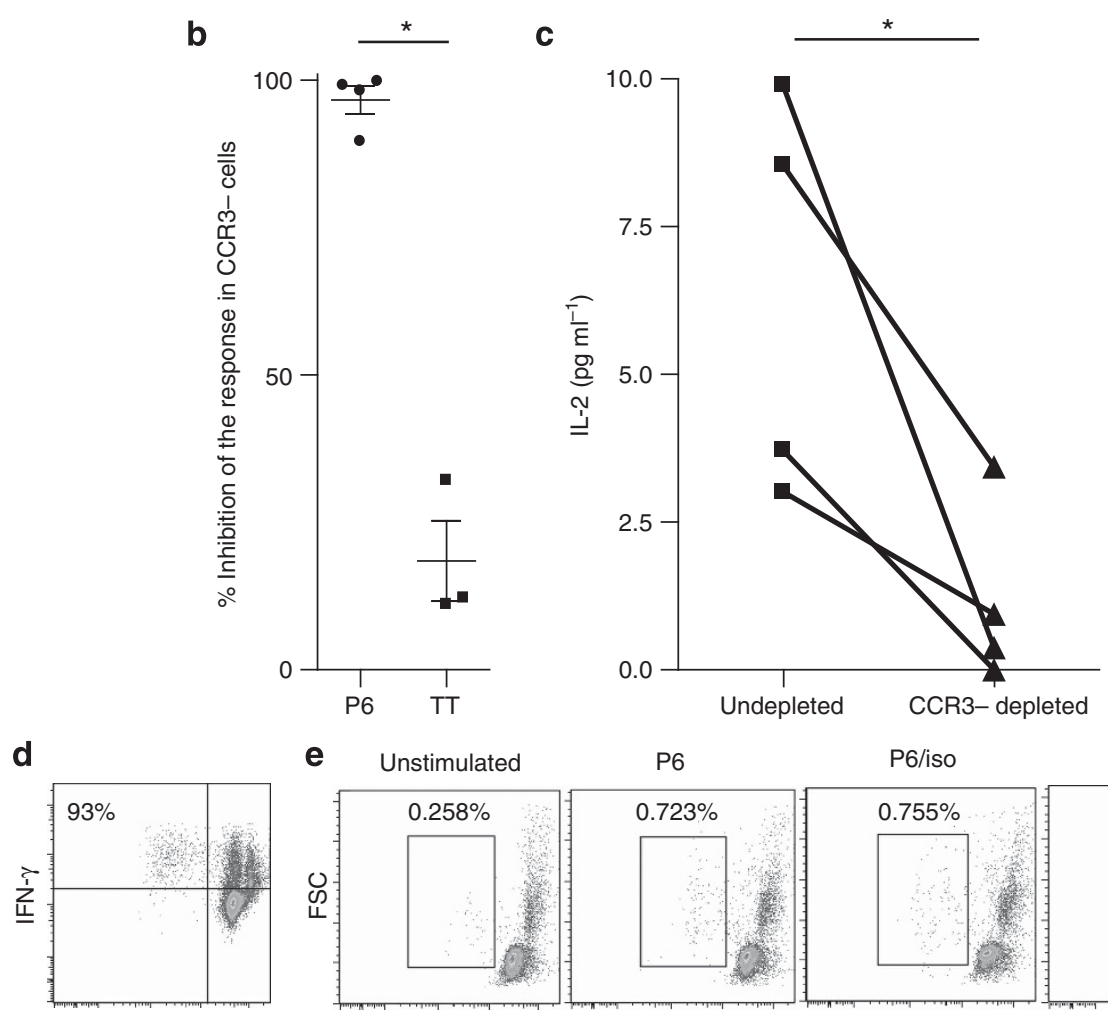

e Unstimulated

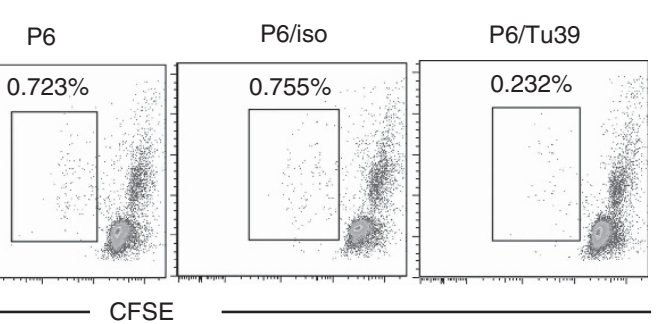

Figure 4 Depletion of $\mathrm{CCR}^{+}{ }^{+} \mathrm{CD} 4{ }^{+} \mathrm{T}$ cells abrogates the $\mathrm{H}$. influenzae-dependent proliferative T-cell response. (a) Carboxyfluorescein succinimidyl ester (CFSE)-labeled peripheral blood CD4 ${ }^{+} \mathrm{T}$ cells, untouched or depleted of CCR3 ${ }^{+}$cells were incubated for 6 days with autologous irradiated peripheral blood mononuclear cell (PBMCs) with or without $H$. influenzae outer membrane protein P6 (P6, top row) or tetanus toxoid (TT, bottom row). Percentage of proliferating CD4 ${ }^{+} \mathrm{T}$ cells was measured by flow cytometry by means of CFSE dilution. (b) Compiled percentage of inhibition of proliferation by $\mathrm{CCR}^{+}{ }^{+} \mathrm{CD} 4^{+} \mathrm{T}$-ell removal from 4 donors is shown. (c) Corresponding IL-2 secretion measured in the supernatants of P6-stimulated cultures with or without CCR $3+\mathrm{CD} 4+\mathrm{T}$ cells depleted $\left({ }^{\star} P<0.05\right)$. (d) CFSE-labeled PBMCs stimulated with $\mathrm{P} 6$ protein for 6 days were analyzed for IFN $\gamma$-production following phorbol 12-myristate 13-acetate/ionomycin-stimulation in the presence of Brefeldin A. The percentage indicated shows percentage of IFN- $\gamma+$ cells among proliferating CD4 + T cells (range 89-95\%, $n=3$ ) (e) CFSE-labeled PBMCs stimulated with P6 protein for 6 days, and incubated with an HLA class II-specific blocking antibody (Tu39) or an isotype-matched control. Data shown are representative of five responding donors.

absence of inflammation, and CCR $4^{+}$cells constitute a minor T-cell fraction in healthy nasal mucosa (Supplementary Figure S3a) as described by others. ${ }^{29}$ We did find that CXCL5 was expressed in the nasal mucosa at steady state, but not in the skin or intestinal mucosa. Studies have suggested a role for the CXCL5 receptor CXCR1 in T-cell trafficking to the nasal mucosa. ${ }^{21}$ However, we found no increase in the proportion of nasal mucosa-derived $\mathrm{CD}^{+} \mathrm{T}$ cells expressing CXCR1 compared with peripheral blood.

Accumulating evidence supports the idea that $\mathrm{T}$ cells acquire trafficking potentials to peripheral tissues following activation in the regional lymphoid tissues, thus ensuring homing of memory 
$\mathrm{T}$ cells with specificities for antigens encountered in that anatomical region. ${ }^{3}$ This concept likely extends to the lower airways, as it was recently shown that human lung tissue contain large numbers of influenza-specific memory $\mathrm{T}$ cells. ${ }^{30}$ Moreover, influenza-specific $\mathrm{T}$ cells exhibited a much reduced frequency in the circulation, and were undetectable in the skin. In the upper airways, NTHi likely represents a frequently encountered pathogen, colonizing up to $75 \%$ of normal adults. ${ }^{15}$ Although direct demonstration of NTHi-specific T cells residing in tissues is lacking, it is reasonable to assume that the specific memory $\mathrm{T}$ cells would recirculate primarily through the upper airways, including the nasal mucosa. Here, we show that the P6-dependent proliferation of peripheral blood $\mathrm{CD}^{+}{ }^{+} \mathrm{T}$ cells was completely abrogated by depleting $\mathrm{CCR} 3^{+}$cells. This finding indicates that the NTHi-reactive $\mathrm{CD}^{+}{ }^{+} \mathrm{T}$ cells are confined to the CCR3-expressing subset and adds further support to the notion that CCR3 is involved in memory T-cell trafficking to the upper airway mucosa. Moreover, our study argues that intranasal vaccine for NTHi could be an attractive approach to induce protective immunity. Interestingly, tissueresident $\mathrm{T}$ cells isolated from the lung were reportedly negative for CCR $3,{ }^{30,31}$ indicating that CCR3 targets T-cell trafficking to the upper airways rather than the lung parenchyma.

CCR3 expression on $\mathrm{CD} 4{ }^{+} \mathrm{T}$ cells has been suggested to be a marker of Th2-polarized memory $\mathrm{T}$ cells, being preferentially expressed on Th2 lines generated under specific culture conditions. ${ }^{32}$ However, analysis of freshly isolated $\mathrm{T}$ cells revealed that the CCR $3^{+}$population contains about equal frequencies of Th 1 and Th 2 cells, and CCR 3 is expressed by less than $5 \%$ of committed Th2 cells in the circulation. ${ }^{33}$ We found that CCR3 + CD4 $+\mathrm{T}$ cells were homogenously positive for the transcription factor T-bet (Supplementary Figure S3b), which is critical for Th1-cell differentiation, and a large fraction of the CCR3 + cells also coexpressed CXCR3, which has been associated with Th1 cells. In fact, our preliminary studies of cytokine production by nasal mucosal $\mathrm{T}$ cells revealed that the vast majority of $\mathrm{T}$ cells were Th1, or to a lesser extent Th17 polarized, whereas Th2 cells were hardly detectable at steady state (Hoffmann et al. in preparation). Moreover, the association between CCR $3^{+} \mathrm{T}$ cells and human allergic disease is controversial; analysis of lung $\mathrm{T}$ cells from asthmatics revealed no difference in CCR3 expression compared with healthy controls. ${ }^{31,34}$ However, one study reported increased numbers of $\mathrm{CD}^{+}{ }^{+} \mathrm{T}$ cells expressing CCR3 in the peripheral blood of allergic rhinitis patients, ${ }^{35}$ which was reduced following allergen immunotherapy. Furthermore, stimulation of CCR3depleted $\mathrm{CD}_{4}{ }^{+} \mathrm{T}$ cells from these patients with allergen resulted in reduced IL-5-production, thus lending further support to the notion that the $\mathrm{CCR} 3^{+}$T-cell population contains antigen specificities relevant to the upper airways.

In conclusion, our findings suggest that CCL28, produced in the nasal mucosa, is presented by the local vascular endothelium and participate in the steady-state homing of CCR3 ${ }^{+}$ $\mathrm{CD}^{+} \mathrm{T}$ cells. This suggests that CCR3 may serve as a candidate molecule to target $\mathrm{CD} 4^{+} \mathrm{T}$ cells operating in the upper airway mucosa.

\section{METHODS}

Subjects. Nasal mucosa prepared for flow cytometry and immunohistochemistry was obtained from the lower edge of the inferior turbinate during surgery for septum deviation. In all cases, the mucosa appeared macroscopically normal and none of the donors had any history of allergic disease ( $n=38$, age 19-51 years old, 16 women). Control tissue from duodenal mucosa was obtained from patients ( $n=3$, age 21,50 , and 75 years, all women) during gastroendoscopic examination for undefined stomach pain. Clinical workup and histopathological examination revealed no signs of gastrointestinal disease in any of the patients. Macroscopically, normal skin samples were obtained by means of punch biopsies $(2 \mathrm{~mm})$ from healthy individuals $(n=3$, age 57,63 , and 70 , all women) undergoing plastic surgery for cosmetic reasons. Peripheral blood mononuclear cells (PBMCs) were obtained from healthy blood donors.

All patients gave their written informed consent, and the study was approved by the Norwegian Regional Committee for Medical Research Ethics.

Tissue preparation and gene expression analysis. Total RNA was extracted with TRI Reagent (Sigma-Aldrich, St Louis, MO) and an RNeasy mini kit (Qiagen, Hilden, Germany). Reverse transcription of RNA was performed with an $\mathrm{RT}^{2}$ First Strand Kit (SABiosciences, Frederick, MD). For analysis of chemokine transcripts, $\mathrm{RT}^{2}$ Profiler PCR arrays (all from SABiosciences) were designed to cover most characterized human chemokines (Supplementary Table S1). PCR was performed according to the manufacturer's protocol, and normalized threshold cycle $\left(C_{t}\right)$ value for each gene, $\left(\Delta C_{t}\right)$, was determined by subtracting the mean $C_{\mathrm{t}}$ value of the housekeeping genes from the individual or mean $C_{t}$ value of the gene of interest. Normalized mRNA expression level was calculated as $2^{(-\Delta C t)}$.

Immunohistochemistry. Tissue preservation and processing was performed essentially as described. ${ }^{5}$ To detect CCL28, we utilized clone 3B1 (mouse IgG1, AbD Serotec, Oxford, UK) or goat IgG (AF717, R\&D Systems, Oxon, UK). CXCL5 was detected using clone S5 (mouse IgG1, ABGENT, San Diego, CA). To detect von Willebrand factor (vWf), rabbit IgG (A0082, DakoCytomation, Glostrup, Denmark) was applied. Irrelevant isotype- and concentration-matched primary antibodies served as negative controls, and Alexa-conjugated secondary antibodies were from Life Technologies (Paisley, UK). Microscopy was performed with an Olympus FV1000 confocal microscope (Olympus, Hamburg, Germany).

FACS analysis. Surgical specimens from healthy donors were finely minced with scissors and incubated with $1 \mathrm{mg} \mathrm{ml}^{-1}$ collagenase (Sigma-Aldrich). Identically treated PBMCs and mechanically disrupted tissue specimens served as controls for the collagenase sensitivity of epitopes. Dispersed cells were stained with the following antibody conjugates: CCR3-APC (clone 61828, R\&D Systems), CCR10-APC (clone 314305, R\&D Systems), CD3-FITC (clone UCHT1, Diatec, Oslo, Norway), CD4-PacificBlue (clone OKT4, eBioscience, San Diego, CA), CLA-FITC (clone HECA-452, BD Bioscience, Franklin Lakes, NJ), CXCR1-PE (clone 5A12, BD Bioscience), and CXCR2-PE (clone 6C6, BD Bioscience). Unconjugated anti- $\alpha 4 \beta 7$ integrin (clone ACT-1) was generously provided by Andrew Lazarovits, and detected with anti-mouse IgG1-PE (Southern Biotech, Birmingham, AL). Propidium iodide was used to exclude dead cells. Fluorescence-activated cell sorting (FACS) was performed on a BD LSR II (BD Biosciences), and analyzed using FlowJo 7.6.3 software (Three Star, Ashland, OR).

T-cell proliferation experiments. $\mathrm{CD} 4^{+} \mathrm{T}$ cells were purified from PBMCs by negative isolation with Dynabeads followed by labeling with $1.5 \mu \mathrm{M}$ carboxyfluorescein succinimidyl ester (CFSE; both from Life Technologies). After staining with anti-CCR3-APC (clone 61828, $\mathrm{R} \& \mathrm{D}$ Systems), CCR $3^{+} \mathrm{CD} 4{ }^{+}$T cells were depleted by FACS sorting with a BD FACSAria (BD Biosciences), after which CCR3-staining of 
CD4 + T cells was indistinguishable from isotype control staining (not shown). CCR3-depleted and undepleted CD $4{ }^{+}$T cells were incubated for 6 days with $50 \mu \mathrm{g} \mathrm{ml}^{-1} \mathrm{H}$. influenzae outer membrane protein $\mathrm{P}^{36}$ or $30 \mu \mathrm{g} \mathrm{ml}^{-1}$ Tetanus toxoid (Calbiochem, Darmstadt, Germany) as control antigen. To ensure that $\mathrm{T}$-cell proliferation was not affected by inadvertent depletion of minor populations of antigen presenting cells expressing CCR3, T-cell fractions were cultured with irradiated autologous PBMCs labeled with CellTracker Violet (Life Technologies) as antigen presenting cells. Cytokines secreted in the supernatants were measured with a Bio-Plex Human Cytokine Assay (Bio-Rad Laboratories, Hercules, CA) as described by the manufacturer. Six independent donors were enrolled in the study and four of them exhibited a proliferative response to P6 stimulation. For the tetanus toxoid experiments, three donors with known vaccination history were recruited. To examine cytokine production in T cells, CFSE-labeled PBMCs from donors with previously established P6-response $(n=3)$ were stimulated for 6 days with $50 \mu \mathrm{g} \mathrm{ml}^{-1} \mathrm{P} 6$ protein, and then stimulated for $3.5 \mathrm{~h}$ with $1.5 \mathrm{ng} \mathrm{ml}^{-1}$ phorbol 12 -myristate 13 -acetate and $1 \mu \mathrm{g} \mathrm{ml}^{-1}$ ionomycin, with $10 \mu \mathrm{g} \mathrm{ml}^{-1}$ Brefeldin A (all from Sigma-Aldrich) added after $1 \mathrm{~h}$ of stimulation. Cells were stained with Fixable Viability Dye eFluor 450 (eBioscience), followed by surface staining for CD3 and CD4. To detect intracellular cytokines, cells were treated with cytofix/cytoperm (BD Biosciences) and stained with antiIFN- $\gamma$-BV510 (Biolegend, San Diego, CA). To assess whether T-cell proliferation was human leukocyte antigen (HLA) class II dependant, CFSE-labeled PBMCs from seven individual donors (of which five exhibited a proliferative response to P6 stimulation) were stimulated for 6 days with $50 \mu \mathrm{g} \mathrm{ml}^{-1} \mathrm{P} 6$ protein together with a blocking monoclonal antibody to human HLA-DR, DP and DQ (Tu39, BD Biosciences) or a control mouse IgG2a antibody (UPC10, SigmaAldrich). Antibodies were refreshed after 3 days of culture.

Statistical analysis. $P$-values were calculated by the GraphPad Prism 4 software (GraphPad Software, La Jolla, CA) using unpaired $t$-tests. An asterisk in the figures represents $P \leqslant 0.05$.

Supplementary Material is linked to the online version of the paper at http://www.nature.com/mi

\section{ACKNOWLEDGMENTS}

We thank Kathrine Hagelsteen, Aaste Aursjø and Lee Hazell for their excellent technical assistance. This study was supported by grants from the South-Eastern Norway Regional Health Authority and the Norwegian Research Council through its Centres of Excellence funding scheme, project number 179573.

\section{DISCLOSURE}

The authors declare no conflict of interest.

c) 2015 Society for Mucosal Immunology

\section{REFERENCES}

1. Jahnsen, F.L., Farstad, I.N., Aanesen, J.P. \& Brandtzaeg, P. Phenotypic distribution of Tcells in human nasal mucosa differs from that in the gut. $A m$. J. Respir. Cell Mol. Biol 18, 392-401 (1998).

2. Masopust, D. \& Schenkel, J.M. The integration of T cell migration, differentiation and function. Nat. Rev. Immunol. 13, 309-320 (2013).

3. Sigmundsdottir, H. \& Butcher, E.C. Environmental cues, dendritic cells and the programming of tissue-selective lymphocyte trafficking. Nat. Immunol. 9, 981-987 (2008)

4. Bromley, S.K., Mempel, T.R. \& Luster, A.D. Orchestrating the orchestrators: chemokines in control of T cell traffic. Nat. Immunol. 9, 970-980 (2008).

5. Johansen, F.E. et al. Regional induction of adhesion molecules and chemokine receptors explains disparate homing of human B cells to systemic and mucosal effector sites: dispersion from tonsils. Blood 106, 593-600 (2005).
6. Kunkel, E.J. \& Butcher, E.C. Plasma-cell homing. Nat. Rev. Immunol. 3, 822-829 (2003).

7. Cuburu, N. et al. Sublingual immunization induces broad-based systemic and mucosal immune responses in mice. Vaccine 25, 8598-8610 (2007).

8. Takamura, S. et al. The route of priming influences the ability of respiratory virus-specific memory CD8 + T cells to be activated by residual antigen. J. Exp. Med. 207, 1153-1160 (2010).

9. Campbell, J.J. \& Butcher, E.C. Chemokines in tissue-specific and microenvironment-specific lymphocyte homing. Curr.Opin.Immunol. 12, 336-341 (2000).

10. Campbell, J.J. et al. The chemokine receptor CCR4 in vascular recognition by cutaneous but not intestinal memory T cells. Nature 400, 776-780 (1999).

11. Soler, D., Humphreys, T.L., Spinola, S.M. \& Campbell, J.J. CCR4 versus CCR10 in human cutaneous TH lymphocyte trafficking. Blood 101, 1677-1682 (2003).

12. Kunkel, E.J. et al. Lymphocyte CC chemokine receptor 9 and epithelial thymus-expressed chemokine (TECK) expression distinguish the small intestinal immune compartment: epithelial expression of tissue-specific chemokines as an organizing principle in regional immunity. J. Exp. Med. 192, 761-768 (2000).

13. Koelle, D.M. et al. Expression of cutaneous lymphocyte-associated antigen by $\mathrm{CD} 8(+) \mathrm{T}$ cells specific for a skin-tropic virus. J. Clin. Invest 110, 537-548 (2002).

14. Rott, L.S. et al. Expression of mucosal homing receptor alpha4beta7 by circulating CD4 + cells with memory for intestinal rotavirus. J. Clin. Invest. 100, 1204-1208 (1997).

15. Erwin, A.L. \& Smith, A.L. Nontypeable Haemophilus influenzae: understanding virulence and commensal behavior. Trends Microbiol. 15, 355-362 (2007).

16. Murphy, T.F., Bakaletz, L.O. \& Smeesters, P.R. Microbial interactions in the respiratory tract. Pediatr. Infect. Dis. J. 28, S121-S126 (2009).

17. de Bree, G.J. et al. Characterization of CD4 + memory T cell responses directed against common respiratory pathogens in peripheral blood and lung. J. Infect. Dis. 195, 1718-1725 (2007).

18. Pan, J. et al. A novel chemokine ligand for CCR10 and CCR3 expressed by epithelial cells in mucosal tissues. J. Immunol. 165, 2943-2949 (2000).

19. Wang, W. et al. Identification of a novel chemokine (CCL28), which binds CCR10 (GPR2). J. Biol. Chem. 275, 22313-22323 (2000).

20. Zlotnik, A. \& Yoshie, O. The chemokine superfamily revisited. Immunity 36 , 705-716 (2012).

21. Francis, J.N. et al. CXCR1 $+\mathrm{CD} 4+\mathrm{T}$ cells in human allergic disease. J. Immunol. 172, 268-273 (2004).

22. Epton, M.J., Hales, B.J., Thompson, P.J. \& Thomas, W.R. T cell cytokine responses to outer membrane proteins of Haemophilus influenzae and the house dust mite allergens Der 11 in allergic and non-allergic subjects. Clin. Exp. Allergy 32, 1589-1595 (2002).

23. Baekkevold, E.S. et al. The CCR7 ligand elc (CCL19) is transcytosed in high endothelial venules and mediates T cell recruitment. J. Exp. Med. 193, 1105-1112 (2001).

24. Carlsen, H.S., Haraldsen, G., Brandtzaeg, P. \& Baekkevold, E.S. Disparate lymphoid chemokine expression in mice and men: no evidence of CCL21 synthesis by human high endothelial venules. Blood 106, 444-446 (2005).

25. Kunkel, E.J. et al. CCR10 expression is a common feature of circulating and mucosal epithelial tissue IgA Ab-secreting cells. J. Clin. Invest. 111, 1001-1010 (2003).

26. John, A.E., Thomas, M.S., Berlin, A.A. \& Lukacs, N.W. Temporal production of CCL28 corresponds to eosinophil accumulation and airway hyperreactivity in allergic airway inflammation. Am. J. Pathol. 166, 345-353 (2005).

27. Panina-Bordignon, P. et al. The C-C chemokine receptors CCR4 and CCR8 identify airway $T$ cells of allergen-challenged atopic asthmatics. J. Clin. Invest. 107, 1357-1364 (2001).

28. Pilette, C., Francis, J.N., Till, S.J. \& Durham, S.R. CCR4 ligands are up-regulated in the airways of atopic asthmatics after segmental allergen challenge. Eur. Respir. J. 23, 876-884 (2004).

29. Banfield, G. et al. CC chemokine receptor 4 (CCR4) in human allergeninduced late nasal responses. Allergy 65, 1126-1133 (2010). 


\section{ARTICLES}

30. Purwar, R. et al. Resident memory T cells (T(RM)) are abundant in human lung: diversity, function, and antigen specificity. PLoS.One 6, e16245 (2011).

31. Campbell, J.J. et al. Expression of chemokine receptors by lung Tcells from normal and asthmatic subjects. J. Immunol. 166, 2842-2848 (2001).

32. Sallusto, F., Mackay, C.R. \& Lanzavecchia, A. Selective expression of the eotaxin receptor CCR3 by human T helper 2 cells. Science 277, 2005-2007 (1997).

33. Kim, C.H. et al. Rules of chemokine receptor association with $\mathrm{T}$ cell polarization in vivo. J. Clin. Invest. 108, 1331-1339 (2001).
34. Morgan, A.J. et al. IL-4-expressing bronchoalveolar T cells from asthmatic and healthy subjects preferentially express CCR 3 and CCR 4. J. Allergy Clin. Immunol. 116, 594-600 (2005).

35. Francis, J.N., Lloyd, C.M., Sabroe, I., Durham, S.R. \& Till, S.J. Tlymphocytes expressing CCR3 are increased in allergic rhinitis compared with non-allergic controls and following allergen immunotherapy. Allergy 62, 59-65 (2007).

36. Hales, B.J. et al. Antibacterial antibody responses associated with the development of asthma in house dust mite-sensitised and non-sensitised children. Thorax 67, 321-327 (2012). 